\title{
New vaccine tailored to the weakened elderly immune system
}

The recent H1N1 swine flu epidemic has been particularly lethal for youngsters, but typical flu outbreaks cause more deaths among the old than the young. In light of this, the US Food and Drug Administration (FDA) approved a seasonal flu vaccine in late December specifically designed to protect people age 65 and over. The vaccine, which will be available for next fall's flu season, comes on the heels of studies showing limited effectiveness of the conventional flu shot among the aged.

The US Centers for Disease Control and Prevention (CDC) currently recommends flu vaccination for people over 65 , who they estimate account for $90 \%$ of influenza-related deaths. But it's well known among vaccine developers that the immune system loses power with age, though exactly why remains a mystery.

Fluzone High-Dose, made by Sanofi Pasteur, contains four times as much of the active antigen component as a conventional shot-thereby eliciting a stronger antibody response in the elderly than do regular vaccines (J. Infect. Dis. 200, 172-180, 2009). Even though higher-titer antibody responses often correlate with protection against disease, the FDA has called on the company to conduct a post-marketing controlled clinical trial asking how well the supershot compares to conventional vaccine in preventing flu.
It's been tough to measure how the decline of the immune system in one's golden years affects the response to vaccines, given the ethics of testing a placebo against a recommended shot. Only one large-scale, placebo-controlled clinical trial of flu vaccine in the elderly has been conducted, and it suggested that only about half of people age 60 and over who received a flu shot were protected against the flu (J. Am. Med. Assoc. 272, 1661-1665, 1994).

Epidemiological studies over the years have arrived at conflicting conclusions. In a recent entry, Roger Baxter, co-director of Kaiser Permanente Vaccine Study Center in Oakland, California and his colleagues reported that vaccination reduces deaths from flu in people age 65 and over by about half. Flu shots prevent one death per 4,000 elderly people vaccinated, they concluded (Am. J. Epidemiol. 170, 650656, 2009).

But Anthony Fiore, a CDC flu epidemiologist, hesitates to draw sweeping conclusions from such epidemiological studies, noting "we need a better way of figuring vaccine effectiveness in older persons."

Increasing the dosage of vaccines, as with Fluzone High-Dose, is only one way to make vaccines more effective among the elderly. Another way is to add adjuvants, substances that boost the immune response. That's the approach taken by Novartis, which received a license in 2000 to market a seasonal flu

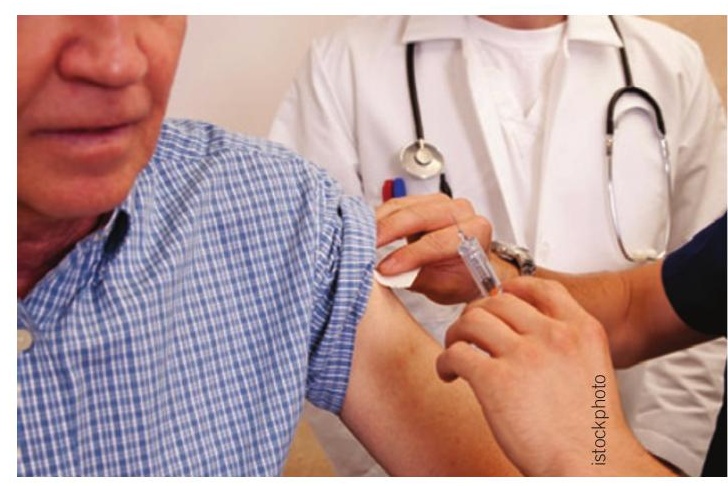

The old guard: New jab promises to protect past one's prime

vaccine in Europe called Fluad, which uses a new adjuvant called MF59, particularly for the elderly.

Novartis has been discussing the conditions for US approval of Fluad with the FDA since 2006, according to Rino Rappuoli, head of research for Novartis Vaccines in Siena, Italy.

Rappuoli notes that similar problems of poor efficacy in the elderly also plague vaccines targeting other diseases. "Influenza is the tip of the iceberg," he says." He imagines that additional vaccines will also be tailored to older people-as well as to other populations with less robust immune systems, such as infants, saying "it's a huge medical need."

Charlotte Schubert, Washington, DC

\section{Mandatory registration required for Korean stem cell lines}

In an effort to rebuild a reputation tainted by disgraced cloning researcher Woo-suk Hwang, the South Korean government is requiring scientists to register all human embryonic stem (ES) cells that are used for research.

As of 1 January, researchers across the country must submit detailed information to the Korea Centers for Disease Control and Prevention (KCDC) in Seoul about all ES cell lines in their labs - whether they derived the cells or not. The necessary documentation includes records of who established the stem cells, a timeline of what the cells have been and will be used for and transfer agreement forms if the lines were purchased.

The registry involves more than just paperwork, however. A KCDC advisory committee will collect the stem cells and analyze them for DNA fingerprints, repetitive gene sequences, karyotype stability and bacterial contamination, among other factors. Adult stem cells do not require registration.

Kim Yong-woo, a senior scientist in the KCDC's division of life science research management, which oversees the registry, notes that the new rules build upon the country's stricter bioethics laws-established in 2005 amidst dispute over whether Hwang obtained the eggs used in his 2004 landmark paper in an ethical manner-to promote the credibility of Korean stem cell research. "At the end of the day, we don't want to have another ethical controversy like the Woo-suk Hwang case," Kim told Nature Medicine.

Currently, only five institutes are authorized by the Korean government to create new stem cell lines-whether derived by cloning or from embryos leftover from fertility treatment-although Soeul's Cha General Hospital and Seoul National University are the only centers distributing their stem cell lines. Another 61 institutes are permitted to conduct research only on established ES cell lines.

Sepill Park, director of the Stem Cell Research Center at Cheju National University, sees the registry as a mixed blessing. He commends the KCDC's efforts to clean up the image of Korean scientists, but says that added incentives are needed to help researchers in the long run.

"It's wonderful that the government is taking initiative with ethical issues," Park says. "But for researchers, we need added benefits to share that information, because we need to keep our competitiveness as well."

D. Yvette Wohn, East Lansing, Michigan 